\title{
Application of Fixed Point Theorem of Convex-power Operators to Nonlinear Volterra Type Integral Equations
}

\author{
Yan Chao-dong \\ Huaiyin Normal University, Huai’an, Jiangsu, 223300, China \\ Copyright (C) 2014 Yan Chao-dong. This is an open access article distributed under the \\ Creative Commons Attribution License, which permits unrestricted use, distribution, and \\ reproduction in any medium, provided the original work is properly cited.
}

\begin{abstract}
This paper firstly generalizes a kind of new operator, i.e. convex-power condensing operator, which was obtained by Sun Jingxian in paper [1], and defines a new class of operator, i.e. $P$-convex-power condensing operator in locally convex space. Also, a new fixed point theorem of this new operator is proved. Finally we apply the results obtained to investigate the existence of solutions for nonlinear Volterra type integral equations in locally convex spaces.
\end{abstract}

Keywords: locally convex spaces; measure of non-compactness; $P$ - convex-power condensing operator; Volterra type integral equations; fixed point theorem

\section{Introduction}

The theory of differential equations, integral equations and integraldifferential equation in abstract space, developing in the first half of the twentieth Century, is a very active research area. Combining the theory of differential equations and functional analysis, using theory and methods of 
functional analysis to study differential equations, integral equations and integral-differential equation in abstract space, it provides a powerful tool for studying the existence of solutions to nonlinear problems in chemistry, physics, biology, economics and other systems.

At present, most of these researches are concentrated on studying the existence of solutions for differential equations, integral equations and integral-differential equations in Banach space. Thus, it has achieved a richer and more exhaustive result. Note worthily, there are much fewer studies about these problems relatively in locally convex spaces, such as [2, 3, 4, 5, 7]. As everyone knows, locally convex space is a class of much wider abstract space and its theory is one of the main contents of nonlinear functional analysis.

Paper [1] defines a new class of operators in Banach space, namely the convex power condensing operator. It also obtains the new fixed point theorem of the operator, which will be applied to solve the existence of global mild solution and positive mild solution, a class with non-compactly, semi-groupedly and semi-linearly developed Equation in space $E$ of Banach.

Inspired by the literature above, the paper firstly generalizes a kind of new operator, i.e. convex-power condensing operator, which was obtained by Sun Jingxian in paper[1], then it defines a new class of operator, i.e. $P$ - convex-power condensing operator in locally convex space. Also, a new fixed point theorem of this new operator is proved. Finally, we apply the results obtained to investigate the existence of solutions for nonlinear Volterra type integral equations in locally convex spaces.

\section{Definition and Lemma}

Before providing the main result, we need to introduce some basic facts about locally convex spaces. We give the definitions as following.

Paper [8] introduced Kuratowski's measure of non-compactness and basic properties of the bounded set in the Banach space.

Definition 1.1 Let $E$ be a Banach space and $S$ is a bounded set in $E$, then $\alpha(S)=\inf \{\delta>0 \mid S$ can be expressed as a finite number of sets and $S=\bigcup_{i=1}^{n} S_{i}$, making

$\left.\operatorname{diam}\left(S_{i}\right) \leq \delta\right\}$ 
as the Kuratowski's measure of non-compactness, non-compactness measure for short, this indicates the diameter of $S_{i}$ for diam $\left(S_{i}\right)$. Obviously, $0 \leq \alpha(S)<+\infty$

Paper [2] gives Kuratowski's measure of non-compactness, determined by the half of its range in locally convex space.

Definition 1.2 Let $X$ be a locally convex space, whose topology generated by these mi-norm family $P=\left\{p_{\alpha}\right\}_{\alpha \in \Gamma}$, noted by $(X, P), \Omega$ is a bounded set in $X$, called $\mu_{p_{\alpha}}(\Omega)=\inf \left\{d>0 \mid \begin{array}{c}\Omega \text { can be expressed as a finite collection union }: \Omega=\bigcup_{j=1}^{n} \Omega_{j} \\ \text { making } \operatorname{diam}_{p_{\alpha}}\left(\Omega_{j}\right) \leq d, j=1,2, \cdots, n\end{array}\right\}$

as $\Omega$ 's non-compactness measure about half of the range of $p_{\alpha}$. Here, $\operatorname{diamp} p_{\alpha}\left(\Omega_{j}\right)$ means $\Omega_{j}$ 's diameter, determined by the half of the range of $p_{\alpha}$. Obviously, $0 \leq \mu_{p_{\alpha}}(\Omega)<\infty$.

Paper [3] explains in detail about Kuratowski's measure of non-compactness in the locally convex space.

As we all know, the famous Schauder fixed point theorem is an important conclusion, extremely widely applied. This conclusion, however, requires operator to be completely continuous, which is a very hard condition. To weaken this condition, we have proposed condensing operator concept. We turn the condition from the completely continuous operator condensing operators to condensing operator, which defined by condensing operators with non-compact measure in the Schauder fixed point theorem.

Definition 1.3 Let $E$ be real Banach space, $D$ is a bounded set of operators in $E$, operator $A: D \rightarrow E$ is called condensing operator. If $A: D \rightarrow E$ is continuous and bounded, and for any non-relatively compact bounded set $S$ in $D$, there is always $\alpha(A(S))<\alpha(S)$.

Paper [1] proposed the new concept of convex-power condensing operator and basically generalizes it. We also apply the Sadovskii fixed point theorem 
to condensing operators further. First, we give you a mark.

Let $E$ be real Banach space, $D \subset E$ is a convex closed set, $A: D \rightarrow D, x_{0} \in D$. For any given $S \subset D$, let

$$
A^{\left(1, x_{0}\right)}(S) \equiv A(S), \quad A^{\left(n, x_{0}\right)}(S)=A\left(\overline{C O}\left\{A^{\left(n-1, x_{0}\right)}(S), x_{0}\right\}\right), n=2,3, \cdots \quad .
$$

Definition 1.4 Let $E$ be real Banach space, $D \subset E$ is a convex closed set, $A: D \rightarrow D$, we calls $A$ the convex-power condensing operator. If $A$ is continuous and bounded, and it has $x_{0} \in D$ and positive integer $n_{0}$, allowing for any non- relative compactness bounded sets $S \subset D$ with $\alpha\left(A^{\left(n_{0}, x_{0}\right)}(S)\right)<\alpha(S)$, of which $A^{\left(n_{0}, x_{0}\right)}(S)$ is defined as (1.3).

Note 1 According to the definition, if $\alpha\left(A^{\left(n_{0}, x_{0}\right)}(S)\right)=\alpha(S), S$ is a relatively compact set in $E$. Clearly, the condensing operator must be cohesive by convex power. What's more, paper [1] establishes a new fixed point theorem about the newly defined convex-power condensing operator, namely the following lemma 2.6 and Lemma 2.7.

Lemma $1.2^{[1]}$ Let $D$ be a nonempty bounded closed convex set of the Banach space, $A: D \rightarrow D$ is convex-power condensing operator, there must be fixed points of $A$ in $D$.

Lemma $1.3^{[1]}$ Let $E$ be real Banach space, $D \subset E$ is a bounded convex closed set, $A: D \rightarrow D$ is continuous. If there is $x_{0} \in D, 0 \leq k<1$, as well as a positive integer $n_{0}$, making $\alpha\left(A^{\left(n_{0}, x_{0}\right)}(S)\right)<k \alpha(S), \forall S \subset D$, there must be fixed points of $A$ in $D$.

Like the notation and definitions of convex-power condensing operator in Banach space of in the paper [1], we give the definition of $P$-convex-power condensing operator in locally convex space.

Let $(X, P)$ be complete Hausdorff locally convex space, $D \subset E$ is a convex closed set, $A: D \rightarrow D, x_{0} \in D$, for any given $S \subset D$, let

$$
A^{\left(1, x_{0}\right)}(S) \equiv A(S), A^{\left(n, x_{0}\right)}(S)=A\left(\overline{c o}\left\{A^{\left(n-1, x_{0}\right)}(S), x_{0}\right\}\right), n=2,3, \cdots
$$


Definition 1.5 If $(X, P)$ is complete Hausdorff locally convex space, $D \subset E$ is a convex closed set, $A: D \rightarrow D$, so $A$ is $P$-convex-power condensing. If $A$ is continuous and bounded, and there is $x_{0} \in D$ and a positive integer $n_{0}$, which makes any non- $P$ - relative compactness of bounded sets $S \subset D$ have $\alpha\left(A^{\left(n_{0}, x_{0}\right)}(S)\right)<\alpha(S) . A^{\left(n_{0}, x_{0}\right)}$ and $(S)$ in it are defined by (1.3).

With the convex Banach space power condensing operators fixed point theorem obtained by paper [1], namely Lemma 2.6, we can get $P$ - convex-power condensing operator's fixed point theorem in locally convex spaces. Because they are proved similarly, the progress is omitted here.

Lemma 1.4 Let $D$ be a non-empty bounded closed convex set in a complete Hausdorff locally convex spaces $(X, P)$, if $A: D \rightarrow D$ is $P$ - convex-power condensing operator, there must be fixed point of $A$ in D.

\section{Existence of Volterra Integral Equations in Locally}

\section{Convex Spaces Nonlinear}

This section examines the existence of

$$
y(t)=h(t)+\int_{0}^{t} g(t, s) f(s, y(s)) d s, t \in J=[0, a]
$$

Nonlinear Volterra integral equations in locally convex spaces. Among them, $h \in C(J, X), f \in C(J \times X, X),(X, P)$ is a complete Hausdorff locally convex space , $R>0$, mark $D=\left\{(t, s) \in R^{2}: 0 \leq s \leq t \leq a\right\}, g \in C(D, R), C(J, X)$ is a collection of continuous images of all slaves from $J$ to $X$. If, $\forall p \in P,\left\{p_{c}\right\}_{p \in P}$ is the family of semi- Fan of $C(J, X)$. Here, $p_{c}(u)=\max _{t \in J} p(u(t))$ and $C(J, X)$ is locally convex spaces by the family of semi- norm. They are noted with $\alpha_{p}, \alpha_{p_{c}}$, which means the measure of 
non-compactness of $X, C(J, X)$ respectively.

Lemma 2.1 ${ }^{[3]}$ ( $\left.a\right)$ Let $H$ be a bounded set of $C(J, X)$, then

$$
\sup _{t \in J} \alpha_{p}(H(t)) \leq \alpha_{p}(H(J)) \leq 2 \alpha_{p_{c}}(H)
$$

Of it, $H(t)=\{x(t) \mid x \in H\}, H(J)=\bigcup_{t \in J}\{x(t) \mid x \in H\}$.

( $b$ ) Let $H$ be a bounded equicontinuous set in $C(J, X)$, we can conclude that

$$
\alpha_{p_{c}}(H)=\sup _{t \in J} \alpha_{p}(H(t))=\alpha_{p}(H(J))
$$

Similar to the proof of Lemma in the paper [1], it is easy to prove the following lemma.

Lemma 2.2 Let $B$ be a bounded equicontinuous subset in $C(J, X)$, $u_{0} \in C(J, X)$, so the same as $\overline{c o}\left\{H, u_{0}\right\}$.

Lemma 2.3 Let $B \in C(J, X)$ be a bounded and equicontinuous, then we can conclude that $\alpha(B(t)) \in C\left(J, R^{+}\right)$, and

$$
\alpha\left(\left\{\int_{t_{0}}^{t} u(s) d s \mid u \in B\right\}\right) \leq \int_{t_{0}}^{t} \alpha(\{u(s) \mid u \in B\}) d s, t \in J .
$$

Lemma 2.4 For any given $R>0, f$ in the $J \times B_{R}$ (of which $\left.B_{R}=\{x \in E: p(x) \leq R, p \in P\}\right)$ is bounded equicontinuous, $H \subset C(J, X)$ is bounded and uniformly continuous, $D=\left\{(t, s) \in R^{2}: 0 \leq s \leq t \leq a\right\}$, $g \in C(D, R)$, we can conclude that $g(t, s) f(s, H(s))$ is also the equicontinuous set in $C(J, X)$.

With the above lemma and obtained fixed point theorem in $P$ - convex-power condensing operator, Lemma 1.3, we can give the existence results of integral equation (2.1). 
Theorem 2.1 Let $f$ meet:

( $H_{1}$ ) For any given $R>0, f$ is in the same row in $J \times B_{R}, p \in P$, $B_{R}=\{x \in X: p(x) \leq R\}$, and there is a continuous function $a(s) \geq 0$ and real numbers $b>0$ making

$$
p(f(s, u(s))) \leq a(s) p(u)+b, \forall s \in J, \forall u \in X,
$$

and $M \int_{0}^{a} a(s) d s<1$. Here, $M=\max \{|g(t, s)|:(t, s) \in D\}$.

$\left(H_{2}\right)$ If there is a constant $L>0$, making for any bounded equicontinuous set $B$ and $t \in J$ in $C(J, X)$, as well as $\forall p \in P$, there is $\alpha(f(t, B(t)) \leq L \alpha(B(t))$. Then the integral equation (2.1) has at least one existent solution.

Proof: Let integral operators $A: C(J, E) \rightarrow C(J, E)$ as follows

$$
(A u)(t)=h(t)+\int_{0}^{t} g(t, s) f(s, u(s)) d s, t \in J
$$

It is easy to prove $A: C(J, E) \rightarrow C(J, E)$ continuous and bounded, and its solution of integral equation (2.1) is equal to the fixed point of operator equations $\quad A \quad$ Let $\quad r \geq\left[p_{c}(h)+M a b\right]\left(1-\int_{0}^{a} a(s) d s\right)^{-1}, \quad$ mark $B_{r}=\left\{u \in C(J, E): p_{c}(u) \leq r\right\}$, then for any $u \in B_{r}$, we can conclude that

$$
\begin{aligned}
p_{c}(A u)(t) \| & \leq p(h(t))+\int_{0}^{t}|g(t, s)| \cdot p(f(s, u(s))) d s \\
& \leq p_{c}(h)+\int_{0}^{t} M\left(a(s) p_{c}(u)+b\right) d s \leq p_{c}(h)+M r \int_{0}^{a} a(s) d s+M a b
\end{aligned}
$$

$\leq r$

With the condition $\left(H_{1}\right)$ and the definition of $A$. Then, $p_{c}(A u) \leq r$, or $A u \in B_{r}$. Therefore, $A: B_{r} \rightarrow B_{r}$ is continuous and bounded. 
Further is the evidence of $A\left(B_{r}\right)$ being the equicontinuous sets in $C(J, E)$. In fact, $\forall u \in B_{r} 0 \leq t_{1} \leq t_{2} \leq a$, we can conclude by the definition of A that

$$
\begin{aligned}
& p\left((A u)\left(t_{1}\right)-(A u)\left(t_{2}\right)\right) \\
& \leq p\left(h\left(t_{1}\right)-h\left(t_{2}\right)\right)+p\left(\int_{0}^{t_{1}} g\left(t_{1}, s\right) f(s, u(s)) d s-\int_{0}^{t_{2}} g\left(t_{2}, s\right) f(s, u(s)) d s\right) \\
& \leq p\left(h\left(t_{1}\right)-h\left(t_{2}\right)\right)+p\left(\int_{0}^{t_{1}}\left[g\left(t_{1}, s\right)-g\left(t_{2}, s\right)\right] f(s, u(s)) d s\right)+p\left(\int_{t_{1}}^{t_{2}} g\left(t_{2}, s\right) f(s, u(s)) d s\right)
\end{aligned}
$$

$\leq p\left(h\left(t_{1}\right)-h\left(t_{2}\right)\right)+\int_{0}^{t_{1}}\left|g\left(t_{1}, s\right)-g\left(t_{2}, s\right)\right|\left(a^{*} r+b\right) d s+M\left(a^{*} r+b\right)\left|t_{2}-t_{1}\right|$.

Of which, $a^{*}=\sup \{a(t) \mid t \in J\}$. And known by the continuity of $h(t)$ and $g(t, s)$, when $\left|t_{2}-t_{1}\right| \rightarrow 0$, the right side of the formula above tends to zero, so $A\left(B_{r}\right)$ is equicontinuous. Let $F=\overline{c o} A\left(B_{r}\right)$, it is obvious that $A$ mapped to $F$, and $A$ is continuous bounded. Known by Lemma 2.4, $F$ is equicontinuous bounded sets in $C(J, E)$.

Then we prove that: $A: F \rightarrow F$ is convex-power condensing operator. Take $u_{0} \in F$, we can prove there is positive integer $n_{0}$, which for any non-relatively compact set $B \subset F, \alpha_{p}\left(A^{\left(n_{0}, u_{0}\right)}(B)\right)<\alpha_{p}(B)$ is right. For any $B \subset F$, we can conclude that $A^{\left(n, u_{0}\right)}(B) \subset B_{r}$ is also bounded equicontinuous from the definition of $A^{\left(n, u_{0}\right)}(B)$ and Lemma 2.2, Lemma 2.4. So for any fixed $n=1,2, \cdots$, with Lemma 2.1, we know that

$$
\alpha_{p}\left(A^{\left(n, u_{0}\right)}(B)\right)=\max _{t \in J} \alpha\left(\left(A^{\left(n, u_{0}\right)}(B)(t)\right), n=1,2, \cdots .\right.
$$

Because of $B$ being a bounded equicontinuous set in $F$ and Lemma 2.1, we know that $\alpha_{p}(B)=\max _{t \in J} \alpha(B(t))$. and with the condition $\left(H_{1}\right)$ and 
Lemma 2.4, we also know that $g\left(t_{1}, s\right) f(s, B(s)), \forall t, s \in J, t \geq s$ is the equicontinuous sets in $C(J, E)$. So added with the condition $\left(H_{2}\right)$ and Lemma 2.3, we can conclude that

$$
\begin{aligned}
\alpha\left(\left(A^{\left(1, u_{0}\right)}(B)\right)(t)\right) & =\alpha((A(B))(t))==\alpha\left(\left\{h(t)+\int_{0}^{t} g(t, s) f(s, B(s)) d s\right\}\right) \\
& =\alpha\left(\left\{\int_{0}^{t} g(t, s) f(s, B(s)) d s\right\}\right) \leq \int_{0}^{t} \alpha(g(t, s) f(s, B(s))) d s \\
& \leq \int_{0}^{t}|g(t, s)| \alpha(f(s, B(s))) d s \leq M L t \alpha_{p}(B)
\end{aligned}
$$

Then due to the degree of continuity and consistency of $A^{\left(1, u_{0}\right)}(B)=A(B)$, Lemma 2.2 and Lemma 2.4, we can know that $g(t, s) f\left(s, \overline{c o}\left\{\left(A^{\left(1, u_{0}\right)}(B)\right)(s), u_{0}(s)\right\}\right)(\forall t, s \in J, t \geq s)$ is equicontinuous too. With Lemma 2.3 and condition $\left(H_{2}\right)$ and (2.7), we conclude that

$$
\begin{aligned}
\alpha\left(\left(A^{\left(2, u_{0}\right)}(B)\right)(t)\right) & =\alpha\left(h(t)+\int_{0}^{t} g(t, s) f\left(s, \overline{c o}\left\{\left(A^{\left(1, u_{0}\right)}(B)\right)(s), u_{0}(s)\right\}\right) d s\right) \\
& \left.=\alpha\left(\int_{0}^{t} g(t, s) f\left(s, \overline{c o}\left\{\left(A^{\left(1, u_{0}\right)}(B)\right)\right)(s), u_{0}(s)\right\}\right) d s\right) \\
& \left.\leq \int_{0}^{t} \alpha\left(g(t, s) f\left(s, \overline{c o}\left\{\left(A^{\left(1, u_{0}\right)}(B)\right)\right)(s), u_{0}(s)\right\}\right)\right) d s \\
& \left.\leq \int_{0}^{t}|g(t, s)| L \alpha\left(\overline{c o}\left\{\left(A^{\left(1, u_{0}\right)}(B)\right)\right)(s), u_{0}(s)\right\}\right) d s \\
& \leq M L \int_{0}^{t} \alpha\left(\left(A^{\left(1, u_{0}\right)}(B)\right)(s)\right) d s \\
& \leq M L \int_{0}^{t} M L s \alpha_{p}(B) d s=M^{2} L^{2} \frac{t^{2}}{2 !} \alpha_{p}(B) .
\end{aligned}
$$

Suppose $\left.\quad k \geq 2, \quad \alpha\left(A^{\left(k, u_{0}\right)}(B)\right)(s)\right)=M^{k} L^{k} \frac{t^{k}}{k !} \alpha_{p}(B), \forall t \in J$, then for $\forall t \in J$,

$$
\alpha\left(\left(A^{\left(k+1, u_{0}\right)}(B)\right)(t)\right)=\alpha\left(\int_{0}^{t} g(t, s) f\left(s, \overline{c o}\left\{\left(A^{\left(k, u_{0}\right)}(B)\right)(s), u_{0}(s)\right\}\right) d s\right)
$$




$$
\begin{aligned}
\leq \int_{0}^{t} \alpha(g(t, s) f(s, \overline{c o} & \left.\left.\left.\left\{\left(A^{\left(k, u_{0}\right)}(B)\right)\right)(s), u_{0}(s)\right\}\right)\right) d s \\
& \left.\leq \int_{0}^{t}|g(t, s)| L \alpha\left(\overline{c o}\left\{\left(A^{\left(k, u_{0}\right)}(B)\right)\right)(s), u_{0}(s)\right\}\right) d s \\
& \leq M \int_{0}^{t} L \alpha\left(\left(A^{\left(k, u_{0}\right)}(B)\right)(s)\right) d s \\
& \leq M L \int_{0}^{t} M^{k} L^{k} \frac{s^{k}}{k !} \alpha_{p}(B) d s=M^{k+1} L^{k+1} \frac{t^{k+1}}{(k+1) !} \alpha_{p}(B)
\end{aligned}
$$

Therefore, by the induction shows that for any $n=1,2, \cdots$, there is

$$
\alpha\left(\left(A^{\left(n, u_{0}\right)}(B)\right)(t)\right) \leq M^{n} L^{n} \frac{a^{n}}{n !} \alpha_{p}(B) .
$$

Thus by the (2.6), (2.10), we know that

$$
\alpha\left(\left(A^{\left(n, u_{0}\right)}(B)\right)(t)\right)=\max _{t \in J} \alpha\left(\left(A^{\left(n, u_{0}\right)}(B)\right)(t)\right) \leq M^{n} L^{n} \frac{a^{n}}{n !} \alpha_{p}(B) .
$$

With $M^{n} L^{n} \frac{a^{n}}{n !} \alpha_{p}(B) \rightarrow 0(n \rightarrow \infty)$, we know that there must be a positive integer to make $M^{n_{0}} L^{n_{0}} \frac{a^{n_{0}}}{n_{0} !} \alpha_{p}(B)<1$, which proves $A: F \rightarrow F$ is a $P$ - convex-power condensing operator. According to Lemma 1.4, there is a fixed point $u^{*}$ of $A$ in $F$, which is the solution of $u^{*}$, the integral equation (2.1) in the $C(J, E)$. Thus the proof is completed.

\section{References}

[1] Sun Jingxian, Zhang Xiaoyan. Convex-power condensing operator's fixed point theorem and its Applications of Abstract Semilinear Evolution Equations [J], Acta Mathematica Sinica,2005,48 (3)439-446.

[2] Polewczak J. Ordinary differential equations on closed subsets of locally convex space with applications to fixed point theorems [J], J Math Anal Appl. 151(1990) 208-225. 
[3] Shi Hongbo, Zhu Jiang. The Existence of Nonlinear Volterra Integral Equations in Locally Convex Spaces [J]. Acta Analysis Functionalis Applicata, 2006, 8(1) 43-50.

[4] Toshio Yuasa. Differential equations in a locally convex space via the measure of nonprecompactness [J], J Math Anal Appl. 84(1981)534-554.

[5] O’Regan D. Integral equations in reflexive Banach spaces and weak topologies [J], Proc Amer Math Soc.1996, 124 (2) 607-614.

[6] O’Regan D. Existence results for nonlinear integral equations [J], J Math Anal Appl.1995 (192) 705-726.

[7] O’Regan D. Some fixed point theorems for concentrative mappings between locally convex linear topological spaces [J], Nonlinear Anal.1996, 12 (27) 1437-1446.

[8] Guo Dajun. Nonlinear Functional Analysis (2nd Edition) M], Jinan: Shan Dong Science and Technology Press, 2001.

[9] Guo Fei, Liu Lishan, Wu Yonghong, Siew Peg-Foo. Global solutions of initial value problems for nonlinear second-order impulsive integro-differential of mixed type in Banach Spaces [J], Nonlinear Anal. 61 (2005) 1363-1382.

[10] Schaefer H.H, Wolff M.P., Topological Vector spaces [M], Berlin: Springer, 1999.

Received: December 11, 2013 